\author{
Historical Studies in Education / Revue d'histoire de l'éducation \\ ARTICLES / ARTICLES
}

\title{
Crétin, Snob et Chic type : figures de l'étudiant dans le journal Brébeuf
}

\section{Par Philippe Garneau}

Université de Montréal

\begin{abstract}
RÉSUMÉ
Fondé dès février 1934, seulement six années après l'ouverture du collège, le journal Brébeufa pour mission, selon sa devise, d'être l' " organe officiel des étudiants du collège Brébeuf ". La lecture du journal Brébeuf permet de connaître les idées, les goûts et, surtout, la perception d'euxmêmes des collégiens issus de l'élite urbaine canadienne-française éduquée par les Jésuites. Elle permet également de faire ressortir l'influence déterminante qu'a exercée le père Rodolphe Dubé, alias François Hertel, sur cette jeunesse instruite. C'est cette représentation de l'étudiant idéal du cours classique que nous allons étudier ici à travers trois types récurrents et intimement liés : le crétin, le snob et le chic type. Objets de vives querelles et de débats animés dès le premier numéro du journal, ces trois types incarnent à la fois les repoussoirs, les inquiétudes et les idéaux de la jeunesse du collège Brébeuf. Des figures d'étudiants peuvent sans doute être trouvées dans d'autres journaux de collèges. Leur étude permettrait de mieux saisir l'identité, ce que l'on nomme alors «l'esprit du collège ", qui a animé les différents établissements classiques et marqué plusieurs de leurs pensionnaires.
\end{abstract}

\section{ABSTRACT}

Brébeuf, the student newspaper of the Collège Jean-de-Brébeuf, was founded in 1934, six years after the opening of the college, and according to its motto, was to be the "official organ of the students of Collège Brébeuf." Reading Brébeuf allows one to be aware of the ideas, the tastes, and particularly the self-perception of students who belonged to the French-Canadian urban social elite, educated by the Jesuit fathers. It also shows the determining influence exercised by Father Rodolphe Dubé, alias François Hertel, on educated youth. This paper will analyze representations of the idealized student of the classical curriculum via three recurring, linked case studies: the cretin, the snob and the chic type. These three case studies, hotly debated within the pages of Brébeuffrom the first issue onward, embody the worries, the ideals and the foils of Brébeuf students. Other case studies can doubtless be found in student publications from classical colleges across Quebec. A comparative study allows us to capture what makes up the identity and the spirit of those establishments. 
Qui est l'étudiant du collège Jean-de-Brébeuf? Archiviste au service de cette institution de prestige pendant cinq années, je me suis souvent posé cette question. C'est dans le journal étudiant, dont j'ai piloté la numérisation et la diffusion en ligne en 2015, que j'ai cherché la réponse. ${ }^{1}$ Nous avons voulu remonter jusqu’à la fondation du collège, en 1928, afin d'assister à la naissance de l'étudiant de Brébeuf. Les premières cohortes sont intéressantes, car ce sont celles qui donnent le ton et qui fixent ce que l'on peut appeler l'esprit du collège. Cet esprit de collège, c'est dans le journal des étudiants que nous pensons le trouver. Nous reprenons en cela l'idée émise par Karine Hébert dans Impatient d'être soi-même : Les étudiants montréalais 1895-1960, selon laquelle : «Les journaux [étudiants] jouent un double rôle : ils marquent l'existence des étudiants comme groupe distinct, en même temps qu'ils contribuent à l'élaboration de son identité. " ${ }^{2}$ La lecture du journal Brébeuf(1934-1972) permet en effet de connaître les idées, les goûts, et surtout, la perception d'eux-mêmes des collégiens issus de l'élite urbaine canadienne-française éduquée par les Jésuites. C'est cette représentation de l'étudiant du collège Jean-de-Brébeuf que nous nous proposons d'étudier ici à travers trois figures récurrentes et intimement liées : le crétin, le snob et le chic type. Les articles " Crétinisme », par Guy Prévost, et " Snobisme intellectuel », d'Antoine Geoffrion, définissent pour ainsi dire ces trois figures dès la première année du journal. Objets de vives querelles et de débats animés, elles incarnent à la fois les repoussoirs, les inquiétudes et les idéaux de la jeunesse du collège durant les années trente.

\section{Le collège sur la montagne}

Les collèges classiques, au-delà de l'enseignement humaniste qui les rassemble, étaient des établissements qui pouvaient être très différents les uns des autres en fonction des objectifs poursuivis par leurs fondateurs, de leur localisation géographique et de l'époque de leur fondation. Dans le cas du collège Jean-de-Brébeuf, que l'on surnomme "le collège de la montagne " en raison de son emplacement près du mont Royal, l'origine de sa création vient du fait que l'ancien Collège Sainte-Marie, situé dans un centre-ville de plus en plus densément peuplé, devenait vraiment trop exigu. Au moment de la première rentrée de septembre 1928, un article du Devoir compare ainsi les deux collèges :

L'un en bas, à travers le bruit, la poussière, la suie et la fumée, s'est taillé par les quatre-vingts années d'efforts, une place enviable au soleil de la vie canadienne; l'autre, sur les hauteurs sereines du mont Royal, dans le grand air de la campagne, vient d'éclore comme un bouton de rose, vient naitre tout d'une pièce, à l'âge d'homme parfait, avec toutes ses dents, le sourire aux lèvres, les yeux irradiants les plus beaux, les plus fermes espoirs de succès. L'un externat, rue Bleury; l'autre internat, à Notre-Dame-des-Neiges; le premier sous le manteau de l'auguste Vierge, Sainte-Marie, le second sous l'égide d'un héraut de l'Évangile : Jean-de-Brébeuf. ${ }^{3}$ 
C'est un collège jésuite - il applique le fameux Ratio Studiorum ${ }^{4}$ —il continue l'œuvre du collège Sainte-Marie et c'est un internat bâti à flanc de montagne, presque à la campagne. À notre connaissance seuls Jacques Beaudry ${ }^{5}$ et Marcel Olscamp ${ }^{6}$ ont effectué des recherches sur les étudiants de ce collège, essentiellement pour y montrer les réseaux qui se sont formés chez les collaborateurs du journal Brébeuf. Il est vrai que ce journal de collège est remarquable par sa longévité — presque toute la période du cours classique à Brébeuf — et par le nombre de personnalités politiques et littéraires qui y sont passées.

La période des années trente y est particulièrement foisonnante, car la rédaction du journal est assurée tour à tour par Paul Gérin-Lajoie (1938-1939), Pierre Elliot Trudeau (1939) et Jacques Lavigne (1940), alors que la direction est, de son côté, assurée par Pierre Vadeboncoeur (1939). À ces étudiants, il faut ajouter l'influence marquante de certains jésuites, dont le père Rodolphe Dubé, alias François Hertel, présent au collège au début et à la fin de cette période.

\section{Le journal Brébeuf: organe officiel du collège Jean-de-Brébeuf}

Fondé en 1934 par des élèves déçus d'être « le seul collège à ne pas avoir son journal ", ${ }^{7}$ Brébeuf livre son dernier numéro en février 1972, année même de l'abandon définitif de la formule du cours classique par le collège Jean-de-Brébeuf. C'est un mensuel de 8 à 16 pages au format in-folio qui paraît d'octobre à mai. Il compte entre 5 et 13 numéros par année. Un numéro coûte 5 cents, un abonnement annuel, $1 \$$ pour les étudiants et $1,25 \$$ pour les non étudiants. La première année, il est tiré à environ 600 exemplaires par numéro. On y trouve bien entendu de nombreuses chroniques liées au collège et aux activités étudiantes : vie sportive, scoutisme, coin des petits, témoignages de finissants et lettres d'anciens. Les productions littéraires des étudiants occupent un espace proportionnel à leur importance dans le cours classique : poésies, contes et pastiches abondent. Mais ce sont surtout les éditoriaux et les chroniques sur la jeunesse et son rôle dans le redressement spirituel et économique du Canada français qui attirent l'attention. Le milieu des années trente voit en effet apparaître la jeunesse en tant que catégorie sociale autonome. Le Manifeste de la jeune génération ${ }^{8}$ des Jeunes-Canada de 1932, appel à " toute la jeunesse de notre race " afin de "reconquérir les positions perdues", est encore dans tous les esprits en 1934 et Brébeufse fait l'écho des manifestations et des publications du mouvement. Mais c'est surtout la Jeunesse étudiante catholique (JEC), fondée en 1935 afin de promouvoir l'apostolat laïc chez les étudiants et rapidement très populaire dans les collèges, qui produit le discours le plus mobilisateur dans les pages du Brébeuf. ${ }^{9}$

\section{Le collège, cette autre famille}

Selon sa devise, le journal Brébeufa pour mission d'être l' " organe officiel des étudiants du collège Brébeuf ". Bien qu'il s'agisse d'une initiative étudiante, on voit bien que la supervision des pères jésuites n'est jamais bien loin : "Je vous regarde faire ", écrit le père recteur Oscar Bélanger S.J. en 1934 en tête du premier numéro. Ce dernier 
décrit ainsi les objectifs du journal étudiant dans un article qui fait figure de premier éditorial : "Qui sait, plusieurs d'entre vous, plusieurs d'entre nous découvriront peut-être dans votre journal la vie réelle du Collège. Votre journal vous fera mieux connaître les uns aux autres et à nous, vos maîtres. "Il n'est pas trop surprenant de voir le recteur insister ainsi sur la vie du collège et sur les liens entre maîtres et élèves; le collège classique, à plus forte raison un collège jésuite, est d'abord conçu comme une communauté éducative. N'oublions pas que la majorité des étudiants de l'époque sont des pensionnaires et que les pères jésuites demeurent sur place, ce qui renforce encore le caractère de " communauté humaine vivante du collège ». ${ }^{10} \mathrm{Il}$ s'agit, selon les concepteurs du cours classique, de créer une communauté favorisant l'émulation et la maturation des esprits; le collège est vu comme une grande famille dont les membres partagent le même goût pour l'héritage grec, la même foi catholique ainsi que les mêmes valeurs humanistes et chrétiennes.

Dans un éditorial publié en septembre 1934, un élève résume parfaitement ce lien idéal entre l'esprit collégial et l'esprit de famille : «Le collège, en théorie, c'est, selon la formule du Père Recteur, "cette autre famille". Grognards ou pas grognards, nous l'admettons, l'esprit collégial à créer doit être analogue à l'esprit de famille : s'amuser ensemble, vivre des mêmes idées, partager les mêmes émotions, accepter la vie avec la même ardeur, s'encourager, être joyeux du même rire. C'est un idéal, et "Brébeuf" fera sentir à tous cet idéal d'esprit collégial. $»^{11}$

Ce discours des étudiants sur la vie de collège — sur eux-mêmes - donnera naissance à un type d'étudiant que les rédacteurs du Brébeuf nomment le " chic type ", figure qu'ils opposent au "snob » et au " crétin " qu'ils jugent sans caractère et incapables de dévouement. Ces figures apparaissent à la faveur d'une querelle sur la valeur de l'éducation au collège qui débute dès le premier numéro et qui s'étend bien au-delà de la première année du journal.

\section{Crétinisme}

C’est Guy Prévost, un étudiant de rhétorique, qui lance la polémique par un article au titre aussi court qu'efficace : "Crétinisme ${ }^{12}$ Cet article est imprimé bien en vue en première page juste sous l'éditorial du père recteur, ce qui laisse à penser que son effet était attendu et calculé. L'article commence ainsi : "Le but principal du cours classique est de nous former le jugement pour apprécier ce qu'il y a de beau et de grand. Bien peu au collège ont cet esprit et il est malheureux de constater que beaucoup d'étudiants sont "épiciers". ${ }^{13}$ C'est ainsi que Prévost qualifie de "crétins " tous ceux qui ne se conforment pas à l'esprit du cours classique. Prévost affirme que cet esprit serait partagé par une minorité d'étudiants; la majorité, "les épiciers ", la " masse au collège ", poursuit-il un peu plus loin, ont des conversations qui sont dignes " de cette bourgeoisie canadienne en "manche de chemise éduquée par une certaine presse" ". Comme on peut le constater, le crétin est assimilé sans ménagement aux figures bien connues du bourgeois et du petit boutiquier, avatars éternels du conformisme, aux préoccupations strictement matérielles et dépourvues d'idéal spirituel. Tout comme le bourgeois, le crétin se distingue par son incapacité à être touché 
par l'art véritable : il lit sans " goûter la pensée de l'auteur ", il méprise "presque complètement la musique " et il ne fait pas la différence entre les verbes " peindre et peinturer ». Ce rapprochement crétin-bourgeois perdure tout au long de la décennie. Nous pouvons résumer ce lien ainsi : le crétin est un étudiant sans idéal qui aspire à devenir un bourgeois sans idéal.

Le crétin est aussi un élève à la personnalité sans relief, inapte à réfléchir par luimême : "Il se contente des formules banales que l'on trouve dans les manuels préférant une pensée livresque à une opinion personnelle. " Alors que le cours classique fait une large place à ce que l'on nomme alors les belles-lettres, l'intérêt du crétin pour celles-ci demeure essentiellement ludique ou utilitaire : le crétin lit " pour savoir comment l'histoire se termine » ou simplement " pour passer quelques examens ». En dehors de ses études, qui ne le préoccupent guère au-delà de l'obtention de son diplôme, le crétin n'a que deux passions : "le hockey " et " les succès mondains ". Nous pouvons dire que, pour le crétin, le cours classique est une fin en soi plutôt qu'une occasion de se former, de développer sa pensée et, surtout, sa personnalité. Le crétin cherche à réussir plutôt qu'à apprendre. Selon l'étudiant Prévost, le crétin se caractérise par son manque de courage intellectuel pour « faire ce travail vraiment formateur, condition essentielle pour produire non seulement des hommes capables de gagner de l'argent, mais aussi des citoyens qui ne resteront pas interloqués devant la moindre question intellectuelle ". Non seulement le crétin manque-t-il de courage-le courage de se remettre en question, dirions-nous — , mais, en plus, il s'affaire à tourner en ridicule tous ceux qui s'efforcent de rehausser le niveau des conversations au collège en les traitant de " "blancs-becs" qui cherchent à épater les autres. » De là à les traiter de snobs, il n'y a qu'un pas que nous allons vite franchir, mais il nous faut d'abord passer par la figure de l'anti-crétin, qui n'est nulle autre que le chic type.

\section{Un chic type très sérieux}

L'article de Prévost paru dans le premier numéro du journal appelait une réponse. Il en reçut une dès le second numéro de la part du père Omer Genest S.J. professeur de rhétorique et modérateur du cercle de l'A.C.J.C. et du cercle Missiologique. Il apparaît que ce dernier a été directement sollicité par Pierre Ranger, le directeur du journal, en ces termes : "On a un grand honneur à vous offrir : un article pour Brébeuf: Conseils pour l'esprit. "Honneur auquel le Père Genest répond : "Un honneur? Non. Mais un piège. " ${ }^{14}$ Le père Genest se méfie en effet de la "polémique [qui] s'est engagée sur le climat intellectuel au Collège ", mais le voilà bien forcé de prendre parti, ainsi il poursuit : "Eh bien, soit! Le crétinisme? Parlons-en avec une franche sincérité. Non pour déplorer ou nier son existence, mais pour esquisser l'étudiant "chic type". " L'objectif du père Genest est de contrer l'article plutôt négatif de Prévost sur le crétinisme au collège en brossant le portrait de son contraire : le chic type. Il s'agit d'un étudiant idéal que Genest place d'emblée sous le patronage de l'écrivain catholique Charles Péguy, dont l'engagement authentique interpelle alors fortement la jeunesse des deux côtés de l'Atlantique. ${ }^{15}$ Comme Péguy, l'étudiant chic type doit " tout prendre au sérieux ", " vivre passionnément " et « devenir un digne 
serviteur de la chrétienté et de son pays ». La figure de Péguy, écrivain par excellence du refus de l'habitude bourgeoise, revient religieusement dans le Brébeuf sitôt qu'il est question de fixer un idéal.

On comprend, bien sûr, qu'avec le père Genest nous sommes bien loin de l'humour potache que l’on attribue généralement aux collégiens. Placé sous le signe de l'idéal, l'exposé du père Genest se cantonne à la dimension scolaire de la vie du collégien : l'étude, le travail intellectuel et la discipline que l'on s'impose à soi-même afin de devenir " un homme cultivé, apte à de grands dévouements ». Le père Genest résume ainsi l'emploi du temps du chic type : "Travail obligatoire de classe, travail libre. "Son chic type a tout du premier de classe sérieux et appliqué qui suit à la lettre l'ensemble des préceptes de l'enseignement jésuite. On pourrait croire cet étudiant tout droit sorti du prospectus publicitaire du collège, qui exigeait en effet de l'étudiant un "minimum de quatre heures de travail par jour, même le dimanche " pour « faire son devoir quotidien, préparer ses classes et compléter sa culture par des études personnelles ". ${ }^{16}$ Dans la plus pure tradition jésuite, l'étudiant chic type du Père Genest favorise "l'assimilation lente " de la matière plutôt que le "bourrage des examens ", il recherche en tout "l'effort personnel [...] qui marque pour toujours un esprit ", il travaille de sa "libre initiative " afin d'étoffer les matières des cours et ses lectures sont "dictées par un goût de sérieux et les réflexions qu'elles inspirent ». S’il lui reste du temps libre après avoir appliqué ce programme rigoureux, l'étudiant chic type le passe à la contemplation, à l'écoute et à la lecture des grandes œuvres, qui " provoquent des admirations enrichissantes ». Naturellement, le chic type a horreur de la médiocrité satisfaite, qui est le propre du crétin; ses années de collège, il les passe à élargir ses horizons intérieurs. Le père Genest est bien conscient que le chic type dont il esquisse ainsi le portrait est un idéal difficile à atteindre pour la majorité des étudiants, qu'il s'agit davantage d'un modèle de dépassement qu'il cherche à imposer. La conclusion de son article ne laisse aucun doute à ce propos : "Étudiant mon frère, ne dis pas que le "chic type" est un idéal de rêve et de chimère. Pour te le décrire, j'ai observé autour de toi et l'ai rencontré. Regarde : Puisses-tu être triste d'en voir si peu! Puisses-tu souffrir de n'en être pas un! »

\section{Snobisme intellectuel}

La figure du snob apparaît dans l'article "Snobisme intellectuel ", ${ }^{17}$ publié en fin d'année scolaire dans le cinquième numéro du journal en juin 1934, c'est-à-dire un peu loin de l'article sur le crétinisme auquel il constitue pourtant, selon nous, la meilleure réplique. L'auteur, Antoine Geoffrion, y trace un portrait général du snob qui reprend presque point par point les arguments utilisés par Guy Prévost pour conclure au crétinisme généralisé au collège. Est déclaré snob celui qui dénigre systématiquement les goûts populaires, le hockey, par exemple, qui méprise les choses concrètes, essentiellement l'argent, et qui adopte une conception esthétisante des arts et des lettres. Les snobs ne cherchent qu'à " montrer leur supériorité sur le commun des mortels " en tenant des discussions élevées sur l'idéal intellectuel ou artistique; ils ne descendent de leur piédestal « que pour regarder avec pitié le pauvre peuple qui n'est 
pas assez formé, pas assez intelligent pour comprendre et goûter de telles choses ". Lorsque les snobs vont au théâtre ou lisent un livre, ce n'est pas pour s'amuser, mais pour " éprouver une jouissance esthétique ou peut-être pour déplorer la bêtise du genre humain ». D'ailleurs, ce n'est pas le livre qu'ils aiment, poursuit Geoffrion, " mais plutôt la sensation de supériorité que leur donnent le pouvoir de comprendre et de goûter une véritable œuvre d'art ". Persuadés d'être les seuls à penser juste, les snobs considèrent "crétins tous ceux qui ne pensent pas comme eux ".

Ces gens-là, écrit Antoine Geoffrion afin de bien marquer le coup dans sa conclusion, «il y en a dans nos collèges bien plus que l'on pense; et on peut même dire qu'ils sont pires que les crétins, car au moins ceux-ci peuvent encore apprendre, tandis que ceux-là sont complètement incorrigibles ». Dans le contexte intra-muros du collège, la référence au crétin ne peut être qu'une pique pas bien méchante, mais une pique tout de même, adressée à Guy Prévost, l'auteur de l'article "Crétinisme ". Selon Geoffrion, le snobisme consiste essentiellement à dénigrer les autres étudiants en les traitants de crétin, exactement ce que l'étudiant Prévost a fait sans vergogne dans le premier numéro du Brébeuf. Le snob agirait ainsi afin d'affirmer sa supériorité sur la masse des étudiants qu'il accuse de ne pas s'intéresser suffisamment aux choses de l'esprit et de nuire au climat intellectuel du collège. Le snob et le crétin sont donc intimement liés : l'un ne peut exister sans l'autre. Le snob a besoin d'une masse de crétins incultes, qu'il désigne lui-même ainsi, pour exister et se distinguer en tant que snob. Le crétin, toujours désigné par les autres, est de son côté le repoussoir idéal qui permet de définir à la fois le snob et le chic type.

En seulement quelques mois, de février à octobre 1934, nos trois types sont bien fixés dans les pages du Brébeuf. ${ }^{18}$ En particulier la figure du crétin, qui s'est imposée dès le premier numéro et qui a, pour ainsi dire, donné naissance aux deux autres. Cette querelle de la première année sur le crétinisme à Brébeuf tourne en fait autour d'un seul et même sujet : l'idéal de l'étudiant du cours classique. Cet idéal nous pouvons facilement le résumer ainsi : éviter les pièges du crétinisme et du snobisme afin de devenir un chic type. Le crétin se satisfait de peu, son seul objectif est de terminer ses études afin de devenir un bon bourgeois. Le snob a un idéal de supériorité intellectuelle; il considère la culture comme une fin alors qu'au collège elle est aussi, et surtout, un moyen pour développer sa spiritualité, sa personnalité et son dévouement. Le chic type seul sait tirer le meilleur parti de son passage au collège et de ce que l'éducation jésuite peut lui offrir. Le chic type est un étudiant qui veut devenir un honnête homme. Contrairement au snob, il s'engage totalement dans ses études afin d'en tirer un bénéfice personnel. Ce bénéfice personnel, il en fera aussi profiter sa nation, car le chic type se distingue du crétin et du snob par son dévouement, et par son engagement sincère et profond dans la société.

Ces trois figures décrivent des étudiants abstraits dont il convient de s'éloigner ou de se rapprocher, mais elles peuvent aussi parfaitement constituer des états successifs chez un même étudiant depuis son entrée au collège, au début de l'adolescence, jusqu'à sa sortie alors qu'il est devenu un homme. N'oublions pas que l'idéal du chic type est le résultat d'un apprentissage et d'un retour sur soi favorisés par l'étude des humanités et des lettres. L'étudiant commence par être un parfait crétin, son 
érudition toute récente le conduit vers un snobisme qu'il arrive néanmoins à maîtriser en devenant un chic type qui-miracle de la pédagogie jésuite-a compris que le véritable idéal est situé hors de soi, dans le dévouement désintéressé. La devise-en français plutôt qu'en latin — dont se dotent les élèves de Rhétorique en 1935 résume parfaitement l'esprit de cet apprentissage : "Devenir ce qu'on est. " ${ }^{19}$ Un roman publié en 1939 met en scène cette métamorphose d'un étudiant moyen vers le chic type : Le Beau Risque ${ }^{20}$ écrit par le jésuite François Hertel, ${ }^{21}$ écrivain qui bénéficie alors d'une immense popularité dans les collèges de la province, plus particulièrement au collège Jean-de-Brébeuf où il a enseigné.

\section{François Hertel, ce chic type}

François Hertel enseigne les belles-lettres au collège Jean-de-Brébeuf en 1931, 1932, 1933, ainsi que la philosophie en 1940. Par son enseignement, mais aussi et surtout par sa personnalité non conformiste, il a durablement marqué les étudiants qui l’ont fréquenté. Pierre Elliot Trudeau en témoigne dans ses mémoires : "Hertel a été pour nous un initiateur exceptionnel dans plusieurs domaines. [...] Les auteurs moins fréquentés, français d'abord, mais aussi anglais, américains, voire scandinaves, c'est Hertel qui me les a fait connaître. ${ }^{22}$ Cette popularité de François Hertel auprès des étudiants a visiblement attiré la suspicion et la jalousie de ses confrères puisque nous avons retrouvé, dans les archives de la Compagnie de Jésus, une lettre dans laquelle le père recteur Oscar Bélanger S.J. prend la défense de François Hertel devant le père supérieur de la compagnie, qui menace carrément de l'expulser des Jésuites. ${ }^{23}$ On reproche entre autres choses à François Hertel d'avoir des "jugements avancés et osés ", "d'être ultra-moderne ", " de pousser à des lectures dangereuses " et d'être "de tendances intellectuelles bien plus profanes que religieuses». Reproches auxquels le père recteur réplique par un éloge de François Hertel professeur : "Il est éminent professeur de Belles-Lettres. Il possède déjà une grande érudition qui lui permet de rendre son enseignement plus intéressant. Il fait aimer la littérature et la composition. Il initie, il donne l'amour des Lettres, à ceux, évidemment qui en sont susceptibles. " Surtout, le père recteur invite le supérieur de la Compagnie à revenir sur sa décision, car « [1] e père Dubé [François Hertel] est trop populaire, au bon sens, et trop aimé de tous ses élèves anciens et nouveaux pour être si proches d'eux. Ceux-ci le visiteraient sans cesse et exprimeraient bien vivement leur surprise de voir que Brébeuf se débarrasse ainsi de ses meilleurs professeurs. "

François Hertel se voit malgré tout contraint de quitter le collège Jean-de-Brébeuf en 1934 pour aller enseigner à Saint-Ignace. Nous pouvons avoir une bonne idée de sa conception de l'enseignement des Belles-Lettres par la lecture du livre que ce dernier a consacré à ce sujet en 1939. La promotion de la lecture, plus particulièrement la lecture des auteurs modernes, est pour François Hertel un aspect primordial de l'enseignement :

Nous aurons beau être éloquents, persuasifs, aimables, fascinateur, si nous ne donnons pas à nos élèves, dès leur année de Belles-Lettres, le goût de la lecture 
(ils devraient l'avoir depuis toujours), un an ne se sera pas écoulé qu'ils seront retombés dans les vieux crétinismes. ${ }^{24}$

François Hertel, nous l'aurons deviné, est pour les élèves du collège la figure de l'anticrétin par excellence. On insère des publicités de ses œuvres dans les pages du Brébeuf, on commente abondamment ses livres et, surtout, on admire son humour et ses traits d'esprit. De son côté, le jeune professeur, il a 26 ans lors de son arrivée au collège, est très proche de ses élèves. Nous pourrions dire que dans " cette autre famille " du collège, François Hertel est un père de substitutions. Son poème "Souvenirs ", dédié à ses anciens élèves, peut nous donner une idée du dévouement et de l'amour sincère du professeur pour ses étudiants :

J'appris auprès de vous comment il faut qu'on aime : ...

Sans espoir de retour,

Comment on trouve là le délice suprême,

Le plus pur des amours.

Je vous aimai pour vous, pour vos âmes ouvertes

Par mes tremblantes mains...

Ensemble, nous faisions tant belles découvertes,

Sur de si beaux chemins ${ }^{25} \ldots$

La parution de son premier recueil de poésie, Les voix de mon rêve, ${ }^{26}$ en 1934 est d'ailleurs accueillie avec enthousiasme par les rédacteurs du journal. C’est Guy Prévost, l'auteur de l'article sur le crétinisme, qui se charge de la recension, il écrit : "L'auteur des "Voix de mon rêve" nous a tracé un magnifique modèle : le tableau du jeune homme idéal, du "chic type" dans les "rêves de jeunesse". Ces rêves, il faut les réaliser. ${ }^{27}$ François Hertel est ainsi assimilé au chic type, à l'anti-crétin, par celui-là même qui a lancé le type du crétin dans les pages du journal. En 1936, Roger Chaput, un étudiant qualifié de " fils spirituel de François Hertel ", ${ }^{28}$ se base sur un article publié par son maître dans L'Action nationale ${ }^{29}$ afin d'aborder le sujet de la littérature canadienne ainsi que le vieux débat concernant son existence. Il y dénonce deux attitudes des étudiants devant les lettres canadiennes : il y a les crétins qui « jugent nos lettres sans en avoir aucune notion ", et il y a les snobs, " qui savent tout et n'ont rien lu ». ${ }^{30}$

Cette association de François Hertel à la lutte contre le crétinisme au collège est particulièrement visible lorsqu'il fonde une Académie ${ }^{31}$ de Belles-lettres en 1934. Les rédacteurs du Brébeuf commentent ainsi la nouvelle :

Triomphe de l'esprit. — l'académie Collégiale fondée par le R.P Dubé, auteur bien connu des "Voix de mon rêve ", tient sa première réunion publique. Cette académie a pour but de combattre le crétinisme sous toutes ses formes et de démentir ceux qui disent que la lecture n'est bonne que pour les poètes, que Poincaré est encore président de la France, que Chateaubriand est surnommé le Génie du Christianisme (ceci est du vécu). Elle démentit aussi ceux qui ne voient au collège que des crétins et des abrutis intellectuels. ${ }^{32}$ 
Deux mois plus tard, un article traite plus longuement des buts poursuivis par l'Académie. ${ }^{33}$ On y fait référence au crétinisme qui empoisonne l'atmosphère du collège et qui serait la cause du "dilettantisme confit en vanité égoïste, insoucieux des intérêts nationaux de notre chrétienté ». Combattre ce crétinisme exige de faire la promotion d'une " culture humaine intégralement catholique " seule à même de préparer l'étudiant à une "vie efficace, active au service de l'église canadienne, de notre pays ». Surtout, on insiste sur l'émulation que créera l'Académie chez l'ensemble des étudiants du collège : "Nous voulons commencer dès maintenant cette vie efficace par un rayonnement d'influence, qui transformera petit à petit notre milieu collégial, qui réalisera en nous d'abord, puis autour, le collégien, l'étudiant "chic type". " L'expression " étudiant chic type " prend ici un sens qui dépasse largement le domaine de la seule réussite scolaire, il s'agit d'un engagement plus profond, d'un engagement de nature spirituelle. Il s'agit, par le travail intellectuel et par la pratique de l'introspection, de « repenser notre christianisme afin que nos âmes ne soient pas des âmes établies, parvenues, bourgeoises ». L'article se termine d'ailleurs par une référence à la revue qui incarne alors ce renouveau spirituel pour une partie de la jeunesse canadienne-française : "Et nous en serons de la relève. " "34

\section{La sérénité du crétin}

En effet, pour les jeunes rédacteurs du Brébeufcomme pour leurs aînés de La Relève, la crise économique est "d'abord métaphysique ". ${ }^{35}$ Issus de milieux favorisés, donc moins touchés par les aspects matériels de la crise, c'est sur le plan moral et spirituel que celle-ci est vécue et abordée par les étudiants du Brébeuf. Les origines de la crise sont recherchées dans le matérialisme, le machinisme, le progrès et l'individualisme de la société moderne. Les solutions proposées tiennent du perfectionnement moral de chacun, de l'apostolat étudiant et de la pratique d'une spiritualité plus authentique. Ces différents thèmes se cristallisent autour de la notion psycholittéraire d'inquiétude, thème alors en vogue des deux côtés de l'Atlantique, dont on peut faire remonter l'origine à la parution, en 1926, de l'ouvrage de Daniel-Rops Notre inquiétude. ${ }^{36}$ En 1936 François Hertel s'en inspire afin d'écrire Leur inquiétude, ${ }^{37}$ livre qu'il destine "à tous les jeunes Canadiens français qui ont du cour ".

L'inquiétude renvoie aux doutes, aux angoisses ainsi qu'aux insatisfactions de la jeunesse instruite. Elle est vécue par eux comme un gage de lucidité. Paul Toupin l'utilise ainsi afin de décrire son camarade Jean Richer : "L'inquiétude intellectuelle qui le tiraille le munit contre tout crétinisme. ${ }^{38}$ Être inquiet c'est refuser le crétinisme, c'est choisir le doute plutôt que le chemin de la facilité, de l'habitude et de la routine. L'œuvre de Charles Péguy, ce "cri d'angoisse ", est au centre de la réflexion des étudiants sur l'inquiétude; "leçon d'héroïsme et d'énergie ", de "goût du risque " et de révolte " contre le monde moderne ", elle est perçue comme un véritable " arsenal à déloger l'habitude de la vie des hommes ". ${ }^{39}$ Refuser l'habitude, c'est d'abord, pour ces étudiants catholiques, refuser de pratiquer une religion de façade qui ne permet pas d'engager tout son être : «La routine vient de notre manque de foi. Et la foi nous manque parce que nous, premièrement avons manqué à la foi ", ${ }^{40}$ écrit Paul Toupin. 
Cette foi renouvelée par l'inquiétude est donc une quête de salut, mais elle est aussi, surtout, un devoir d'agir dans le monde, car " agir c'est vivre ». Par sa capacité de rompre avec l'habitude, l'inquiétude est un adjuvant :

L'action, c'est marcher avec une pensée dans la tête, marcher avec un motif. C'est marcher sincèrement dans le sens de ce motif. L'action, c'est voir clair, haut et loin. C'est casser la croûte de matière qui nous tient dans la grande dalle $\mathrm{du}$ hasard et de la routine où nous coulons lourdement et flasques comme des bons hommes de mercure. ${ }^{41}$

\section{Une réforme personnelle}

L'action, pour les étudiants de Brébeuf de cette époque, cela signifie surtout écrire : écrire pour émouvoir, écrire pour subjuguer, écrire pour convaincre. Apprendre à bien parler et à bien écrire — à faire des discours - est certainement une des grandes finalités d'un cours classique qui vise essentiellement à produire une élite, à former de futurs chefs. Aussi, les appels répétés dans les pages du journal à la refrancisation, à l'apostolat et à la mobilisation fatiguent un peu à la longue. Certains étudiants n'hésitent pas à le faire remarquer. L'un d'eux, Jacques Le Duc, y va d'un fort coup de gueule dans le numéro du 21 mars 1935 :

Action. Action. Action. Articles vertigineusement philosophiques de philosophes insoupçonnés. Conseil quasi-évangélique de princes du débraillé. Puissantes apostrophes nationalistes d'acheteurs-chez-nous qui s'habillent chez Simpson's. Littérature hachées, surponctuées, rissolées, sucées, resurponctuées comme au hasard. Enfantement bi-mensuel de dogmes olivaro-révolutionnaires, religieux, mystiques — oh! la contemplation si reposante!-Coups de plume, coups de mâchoire anti-financiers, anti-anglais, anti-tout. ${ }^{42}$

Cet étudiant en a visiblement assez de ces « messieurs du bel esprit [qui] ne peuvent pas assez ne pas être crétins ». Il propose que ces appels à l'action, qu'il ne juge pas mauvais en eux-mêmes, soient précédés d'une "réforme personnelle ». L'éducation reçue au collège devrait, selon lui, être une " promesse pour plus tard ", car, avant d'agir il faut d'abord penser à " se bâtir ou se rebâtir chacun soi-même ". Paradoxalement, cela constitue encore un appel à la mobilisation, mais à une mobilisation pour soi, afin de former sa personnalité. Il s'agit, en quelque sorte, de faire naître en soi le chic type esquissé par le père Genest. Quelques mois auparavant, un autre élève écrivait d'ailleurs, à ce propos, que « l'action, avant d'être influence, doit être une culture de la personnalité ». ${ }^{43}$

Développer sa personnalité n'est pas chose aisée; le risque de confondre celle-ci avec le snobisme, l'individualisme ou l'égoïsme est grand. L'idée généralement admise au Brébeufest que les années de collège sont déterminantes dans la formation de cette personnalité : "L'étudiant se forme au contact des lettres et des arts en général, une 
opinion qui lui est propre; c'est l'embryon de sa personnalité qui se révèle ", ${ }^{44}$ écrit Yves Prévost dans le numéro du 23 mars 1940. Reprenant les termes de la querelle de 1934 sur le crétinisme, il insiste sur la différence entre la "vraie personnalité ", qui distingue de la masse, et le snobisme, qui est une personnalité demeurée superficielle. Chose curieuse, l'article de Prévost est accompagné d'une note de la rédaction qui tient à spécifier que " la personnalité qu'on veut pour soi-même, pour se distinguer de la masse, frôle dangereusement l'égoïsme ». Il est vrai qu'Yves Prévost défend une conception de la personnalité assez particulière qui, c'est le moins que l'on puisse dire, ne cadre pas vraiment avec le dévouement désintéressé que cherchent à inculquer les jésuites :

Souvent, ceux qui confondent personnalité et snobisme sont ceux qui en possèdent le moins. [...] Si le fait de prendre contact avec la littérature et les arts, de chercher à comprendre des choses difficiles à comprendre, d'en saisir la plus grande partie possible, (il faut faire des concessions...), c'est du snobisme, Eh bien! Je veux être snob. Si le fait d'être vu avec un livre de Claudel, de Péguy et, j'irai même jusqu'à dire, de Valéry, c'est du snobisme, Eh bien! Je consens à être snob. Si le fait d'avoir en général, tout ce qui dépasse le "déjà vu ", c'est du snobisme. Eh bien! Je veux encore une fois passer pour être snob. Si le fait, après son cours, de savoir plus que quelques noms d'auteurs, de musiciens et d'artistes et d'avoir pénétré plus que superficiellement dans leurs œuvres, c'est du snobisme, Eh bien! Qu'on mette le snobisme au programme du cours classique. ${ }^{45}$

\section{Un collège de snobs ou de chics types?}

L'article d'Yves Prévost est une exception. Habituellement, on ne se réclame pas du snobisme dans le Brébeuf. Ne pas passer pour snob est même une préoccupation constante chez les rédacteurs du journal. Nous serions portés à penser que c'est parce que la chose ne va pas de soi qu'elle préoccupe aussi sérieusement une partie des étudiants. Les apprentis journalistes du Brébeuf sont d'ailleurs conscients de ce travers et il leur arrive de plaisanter à ce sujet. Par exemple, au moment de faire des prédictions sur les événements qui marqueront la vie au collège en 1935, l'un d'eux écrit : "Nantel écrira son sixième article sur le snobisme. " ${ }^{46}$ Cela montre bien que la dénonciation du snobisme, en plus d'être un sujet des plus convenus, est considérée comme la spécialité de certains élèves.

Or, le plus souvent, c'est sous couvert de réponse à la critique venant de l'extérieur que les rédacteurs du Brébeuf clament bien haut qu'ils ne sont surtout pas des snobs. La meilleure manière de le faire reste de prouver que le collège n'est pas une citadelle intellectuelle fermée aux débats qui agitent la province en ces temps de crise. Ainsi, à l'occasion d'une campagne de souscription à L'Action nationale, périodique qui est relancé en 1933, un membre de la Jeunesse étudiante catholique écrit : "Évidemment, la jeunesse bouge. Pensez-donc! 60 élèves du collège des snobs qu'avec un malin plaisir, 
on traite de petits gourmands, de mondains, de sportifs enragés, 60 goûtent une revue comme L'Action nationale! Signe des temps! ${ }^{47}$ Deux ans plus tard, l'idée que le temps du snobisme est révolu à Brébeuf est au centre d'un long témoignage, publié sur deux pleines pages, intitulé "Notre cours classique ». L'auteur, Jean Filion, est un finissant de la promotion de 1936, la première à avoir effectué ses huit années de cours classique à Brébeuf. Il affirme que l'origine de cette modification dans l'opinion doit être attribuée à un changement d'esprit chez les étudiants :

Il était un temps où l'on disait avec raison que Brébeuf était un collège de snobs. On le dit encore aujourd'hui en certains milieux, mais avec beaucoup moins de raison. Car c'est un fait qu'une bonne partie des élèves du collège n'ont plus deux opinions : une officielle et l'autre personnelle. Aujourd'hui, elles ont tendance à se confondre, et c'est là la marque du nouvel esprit de Brébeuf : un esprit de franchise et de vérité. ${ }^{48}$

Ce finissant a peut-être péché par optimisme en évoquant ce changement d'opinion. En octobre 1939, une lettre publiée dans le journal étudiant du collège Garnier à Québec nous indique que l'opinion que l'on pouvait avoir des étudiants de Brébeuf à l'extérieur n'a, en fait, pas beaucoup changé. Rédigée par le jeune René Lévesque, qui est alors le directeur du Garnier, cette lettre publique est adressée à son ami du pensionnat de Gaspé, Jean-Paul Bérubé, maintenant étudiant à Brébeuf. René Lévesque lui demande essentiellement de répondre à la question suivante : "Quelle sorte de gens êtes-vous, vous autres de Montréal? " Cela afin de nuancer certains préjugés que des étudiants de Québec entretiennent sur ceux de Montréal :

Nous avons déjà rencontré certains exemplaires de Montréalais; et il y en a, chez nous, qui généralisent ces cas et vous imaginent comme des grands pâlis par l'air rare de la métropole, toujours tirés à quatre épingles, tels des cartes de mode "Au bon goût de... chez Eaton " ... ou encore pour des " farauds " qui pensent tout avoir, " the best in the world ", et qui, bec dédaigneux, font fi de tout ce qui n'est pas extrait de leur Île ${ }^{49} \ldots$

En plus de nous informer sur l'opinion que l'on peut avoir des étudiants de Brébeuf dans un autre collège, cette lettre est intéressante, car elle montre que le journal du collège ne vit pas en vase clos. Des correspondances s'établissent avec les rédacteurs des journaux des autres collèges, souvent, comme c'est le cas ici, dans un esprit de saine compétition entre éternels rivaux. Le journal Brébeuf consacre une pleine page de son numéro du 23 décembre 1939 à répondre à la lettre de René Lévesque. JeanPaul Bérubé concède qu'à son arrivée au collège, il partageait l'opinion de Lévesque, mais que maintenant qu'il les connaît, il trouve que les étudiants de Brébeuf sont « les plus chics types que l'on puisse rencontrer ». Il explique :

La note caractéristique des élèves de Brébeuf — et c'est ce qui porte les gens de l'extérieur à voir en nous du snobisme — c'est que nous sommes foncièrement 
indépendants, pris en groupe ou individuellement. Non pas que nous soyons égoïstes au point que joies et souffrances d'autrui nous laissent indifférents, mais semble-t-il, nous avons fait nôtre le vieux dicton américain : « Mind your own business. " ${ }^{50}$

Cet esprit d'indépendance caractérisant les chics types du collège Jean-de-Brébeuf est mentionné une autre fois, quelque mois plus tard, dans des circonstances similaires. Cette fois c'est Pierre Elliot Trudeau, rédacteur en chef du journal, qui répond à Gérard Pelletier, le président de la JEC. Ce dernier dans un éditorial du journal JEC demande que, "sous le patronage de $J E C$ qui les relie tous, chaque journal étudiant définisse son attitude, par la plume de son responsable étudiant, dans son prochain numéro ${ }^{51} \mathrm{Il}$ s'agit en quelque sorte des premiers préparatifs qui mèneront ultimement à la création par Pelletier de la Corporation des Escholiers Griffonneurs en 1943, fédération de journaux étudiants dont Brébeuf sera membre. La direction du journal, qui n'aime pas particulièrement cette intrusion de la JEC dans ses affaires, charge le jeune Trudeau de la responsabilité de composer un texte que nous pouvons qualifier de réponse de jésuite, ou de politicien, tant il ne répond pas à la question posée par Pelletier :

Je vous réponds que «BRÉBEUF » est publié par les élèves du collège. Il ne saurait donc être autre chose que l'expression, évidemment inadéquate, de leur personnalité. Il s'adresse à tous ceux qui veulent payer le prix d'un abonnement afin de connaître cette personnalité. Et il n'exerce que l'influence d'une personnalité. [...] C'était la façon la plus simple de vous faire connaître l'étudiant de Brébeuf. Et je crois qu'il vaut la peine d'être connu, car c'est un chic type; vous n'en trouverez pas de plus passionné de culture, de plus serviable, ni de plus sincère; il aime à penser par lui-même et à dire ce qu'il pense. S'il affecte (quelquefois) d'être frais, c'est qu'il tient à affirmer son indépendance. ${ }^{52}$

\section{Conclusion}

On peut penser qu'il peut être avantageux d'affirmer ainsi son indépendance lorsque l'on appartient à la bourgeoisie urbaine et que huit années de cours classique nous ont transmis l'habitus de l'honnête homme. Nous pourrions même dire que c'est là, dans cette attitude d'indépendance désintéressée attribuée au chic type, que réside l'ultime marque de snobisme. S'il est vrai, comme l'écrit un étudiant en 1942, que "c'est chose respectable que la culture intellectuelle, mais peut-on se prévaloir et se faire un snobisme de ce que l'on a par privilège $",{ }^{53}$ faire comme s'il n'y avait pas de privilège constitue en fait le véritable privilège dont le chic type doit se prévaloir afin d'assurer sa réussite dans le monde. N'oublions pas que, si l'on vante tant le désintéressement des études classiques, c'est surtout parce que ces études conferent alors à celui qui les termine un prestige et des positions de pouvoir qui sont, de leur côté, fortement intéressés. Il serait pertinent de faire une étude comparative entre Brébeufet des journaux 
de collège qui accueillent des étudiants de milieux plus modestes. Cela permettrait de déterminer si les figures d'étudiants mises de l'avant par le journal sont le pur produit du cours classique en général ou un produit particulier de Brébeuf. Il serait également intéressant d'étudier, dans d'autres journaux étudiants, le sentiment d'inquiétude des collégiens des années trente ainsi que la réception des œuvres et de l'enseignement de François Hertel.

Que reste-t-il aujourd'hui de cet esprit de collège mis en place au début des années trente afin de prémunir l'étudiant contre le crétinisme, de le tenir éloigné du snobisme et de former des chics types au dévouement que l'on ose croire désintéressé? Peu de chose sans doute, à l'exception, peut-être, de l'essentiel : la vieille notion d'élite. Bien sûr, cette notion a évolué; on ne mise plus sur la maîtrise des belleslettres afin de distinguer l'homme cultivé, mais plutôt sur le caractère international de la formation et sur la recherche de l'excellence. Le caractère élitiste de l'éducation privée, lui, demeure. Tout dans ce collège est encore marqué du sceau de l'élitisme : mise de l'avant de filières d'excellences, cohortes effectuant sept années au collège, devise en latin, tableau d'honneur bien visible à l'entrée, bourses d'excellences fondées par d'anciens étudiants illustres, mosaïques des cohortes passées décorant les murs de larges corridors carrelés menant à de petites classes toujours munies d'anciennes estrades et, surtout, l'architecture de la façade qui impose le respect et la dignité. Les snobs, les crétins et les chics types, nés dans les pages du Brébeuf en 1934, n’ont certainement pas déserté un endroit qui a su demeurer aussi fidèle à lui-même.

\section{Endnotes}

1 L'ensemble des numéros du journal Brébeufpeut être consulté en ligne sur le site des archives du collège à l'adresse suivante : http://www.brebeuf.qc.ca/bibliotheques/ journal-le-brebeuf/.

2 Karine Hébert, Impatient d'être soi-même : Les étudiants montréalais 1895-1960 (Québec : PUQ, 2008), 54.

3 Édouard Lecompte S.J., "Deux collèges ", Le Devoir, XIX, 206, $1^{\text {er }}$ septembre 1928 : 1.

4 Le Ratio Studiorum fixe dans un code les postulats humanistes ainsi que la structure du curriculum de la pédagogie jésuite. À ce sujet, lire Georges Leroux, «La raison des études. Sens et histoire du Ratio Studiorum ", Études françaises, 31, 2 (1995) : 29-44.

5 Jacques Beaudry et Robert Daneau, Le rébus des revues : petites revues et vie littéraire au Québec (Québec : Presses Université Laval, 1998).

6 Marcel Olscamp, "Un air de famille. Entre La relève et Refus global : la génération cachée ", Tangence, 62 (2000) : 7-33.

7 Le père recteur, "Vous l'avez voulu ", Brébeuf, I, 1, 24 février 1934: 1.

8 Rédigé par André Laurendeau et approuvé par Pierre Dansereau, Pierre Asselin, Roger Larose, Pierre Dagenais, Dollard Dansereau, Paul Dumas et Gérard Filion, le manifeste est lancé au Gésu en 1932 avant d'être reproduit dans plusieurs journaux de l'époque : Le Devoir, L'Action catholique, Le Progrès du Saguenay, Le Quartier Latin, La Presse, Le Canada, Le Droit, La Patrie et Le Béret.

9 Au sujet de la JEC et du rôle qu'elle a joué dans l'émergence de la jeunesse en tant que catégorie sociale autonome, voir Louise Bienvenue, Quand la jeunesse entre en scène (Montréal : Boréal, 2003). 
10 Claude Corbo, Les jésuites québécois et le cours classique après 1945 (Québec : Les éditions du Septentrion, 2004), 97.

11 Philippe Langlois, "Réponse à un point d'interrogation », Brébeuf, II, 1, 26 septembre $1934: 3$.

12 Guy Prévost, "Crétinisme », Brébeuf, I, 1, 24 février 1934: 1.

13 L'expression "épicier » est sans doute une référence à la correspondance, bien connu des collégiens de l'époque, du poète Octave Crémazie avec l'abbé Casgrain : « [...] nous n'avons malheureusement qu'une société d'épiciers. J'appelle épicier tout homme qui n’a d'autre savoir que celui qui lui est nécessaire pour gagner sa vie, car pour lui la science est un outil, rien de plus. " Octave Crémazie, Euvre complète (Montréal : Beauchemin \& Valois, 1882), 28.

14 Omer Genest J.S., "Conseil pour l'esprit », Brébeuf, I, 2, 20 mars 1934 : 1.

15 Jusqu’à la fin des années cinquante Charles Péguy est beaucoup lu dans les collèges classiques. Au sujet de la réception de l'œuvre de Péguy au Canada français, voir Pierre Savard, " Notre Péguy ", Les Cahiers des dix, 45 (1990) : 193-216.

16 Archives du collège Jean-de-Brébeuf, Prospectus publicitaire du collège, 1934.

17 Antoine Geoffrion, "Snobisme intellectuel », Brébeuf, I, 5, 14 juin 1934 : 2.

18 Le bilan de l'année 1934 publié en fin d'année scolaire dans les pages du Brébeuf consiste essentiellement en un résumé des querelles déclenchées dans le collège par les articles sur le crétinisme et le snobisme.

19 Rolland Poirier, "Devenir ce qu’on est », Brébeuf, III, 4, 20 novembre 1935: 1.

20 François Hertel, Le beau risque (Montréal : Édition Bernard Valiquette, 1939).

21 Pseudonyme du Père Rodolphe Dubé. Par souci de clarté nous allons utiliser uniquement le nom de François Hertel. Bien qu'il enseigne sous le nom de Rodolphe Dubé S.J., c'est effectivement à cette époque qu'il commence à être beaucoup plus connu sous son pseudonyme.

22 Pierre Elliott Trudeau, Mémoires politiques (Montréal : Le Jour Éditeur, 1993), 32. Contrairement à une idée reçue, ce dernier n'a jamais été l'élève de François Hertel.

23 Archives des jésuites au Canada, Fonds Collège Jean-de-Brébeuf 1928-1988, GLC, C-0006, C-6-75, Correspondance Rodolphe Dubé, 15 juillet 1934.

24 François Hertel, L'enseignement des Belles-Lettres (Montréal : Aux ateliers de l'entr'aide, 1939), 8.

25 François Hertel, «Souvenirs ", Brébeuf, I, 4, 11 mai $1934: 4$.

26 François Hertel, Les voix de mon rêve (Montréal : Éditions Albert Lévesque, 1934).

27 Guy Prévost, "Sincérité d'un ancien élève ", Brébeuf, II, 2, 13 octobre 1934 : 1. Le poème "Le rêve de jeunesse " dont il est fait mention dans l'article a été publié dans le numéro précédent du Brébeuf. Il est dédié "Aux jeunes de chez nous ». François Hertel y exhorte la jeunesse catholique à réaliser leurs rêves généreux et patriotiques.

28 "Roger Chaput ", Brébeuf, III, 14-15-16, 30 mai 1936: 6.

29 François Hertel, "La littérature canadienne-française (Son rôle dans une éducation nationale) ", L'Action nationale, V, 5, mai 1935 : 277-289.

30 Roger Chaput, "Notre littérature », Brébeuf, III, 13, 2 mai $1936: 1$.

31 La formation d'académies sur le modèle de l'Académie française est une pratique pédagogique courante dans les collèges classiques. Claude Corbo en donne la définition suivante : "Il s'agit de sociétés d'élèves qui se réunissent, après les heures de cours, pour débattre entre eux de questions intellectuelles ou scientifiques, particulièrement dans les classes terminales du programme. La littérature ou la philosophie se prêtent bien à ce genre d'exercice. Dans ces sociétés de discussion et d'échange, le public est formé d'autres élèves. " Claude Corbo, Les jésuites québécois et le cours classique après 1945 (Montréal : Les éditions du Septentrion, 2004).

32 "Année de collège modèle : 1933-34 ", Brébeuf, I, 5, 14 juin 1934 : 3.

33 "Cercle ou académie ?", Brébeuf, II, 1, 26 septembre 1934 : 4. 
34 Fondée en 1934 par Paul Beaulieu, Robert Charbonneau et Claude Hurtubise, tous trois anciens du collège Sainte Marie, La relève est une revue d'inspiration non conformiste qui s'adresse à la jeunesse. On y tient un discours très critique à propos de l'absence de vie spirituelle au Canada français. À ce sujet, voir Stéphanie Angers-Fabre, "Le versant Canadien-français de la génération non conformiste européenne des années trente : la revue La Relève ", Recherches sociographiques, 43, 1, 2002 : 133-148.

35 La Direction, "positions ", La relève, premier cahier, première série (mars 1934) : 3.

36 Daniel-Rops, Notre inquiétude (Paris : Librairie académique Perrin, 1927).

37 François Hertel, Leur inquiétude (Montréal : Éditions Albert Lévesque, 1936). En 1953, un ancien élève du collège Jean-de-Brébeuf publie un livre majeur sur le sujet : Jacques Lavigne, L'inquiétude humaine (Paris : Aubier, Éditions Montaigne, 1953).

38 Paul Toupin, "Le vice-président ", Brébeuf, III, 9-10-11, 15 février 1936 : 9.

39 Charles Lussier, "Charles Péguy ", Brébeuf, VI, 3, 3 décembre $1938: 3$.

40 Paul Toupin, "Nos forces spirituelles », Brébeuf, IV, 6-7-8, 21 décembre $1936: 8$.

41 Gabriel Houde, "Agir, c'est vivre ", Brébeuf, II, 8, 30 janvier 1935: 2.

42 Jacques Le Duc, "Douche froide ", Brébeuf, II, 11, 21 mars 1935 : 2.

43 Gabriel Houde, op. cit.

44 Yves Prévost, «Snobisme ou personnalité », Brébeuf, VII, 6, 23 mars $1940: 2$.

45 Ibid.

46 Jafsie [pseudonyme], "En 1935, rien de nouveau », Brébeuf, 2, 8, 30 janvier 1935: 5.

47 "La jeunesse bouge! ", Brébeuf, II, 10, 2 mars 1935 : 1.

48 Jean Filion, "Notre cours classique », Brébeuf, III, 17, 18 juin $1936: 1,8$.

49 René Lévesque, "Nous ", Le Garnier, octobre 1939.

50 Jean-Paul Bérubé, "Vous de la capitale et nous de la métropole », Le Brébeuf, VII, 3 et 4, 23 décembre 1939: 11.

51 Pierre Elliot Trudeau, "Ceci est l'éditorial », Brébeuf, VII, 8 et 9, 12 juin $1940: 2$.

52 Ibid.

53 André Fortin, «Équilibre », Brébeuf, IX, 6, 27 mars 1942: 1. 